\title{
Association between Inflammatory Bowel Disease and Lactose Intolerance: Fact or Fiction
}

\author{
Mohammad Maysara Asfari, Muhammad Talal Sarmini ${ }^{1}$, Katherine Kendrick², Amit Hudgi, Pearl Uy, \\ Subbaramiah Sridhar and Humberto Sifuentes \\ Department of Gastroenterology, Medical College of Georgia/Augusta University, Augusta, GA; Department of Internal \\ Medicine, Cleveland Clinic ${ }^{1}$, Cleveland, $\mathrm{OH}$; Department of Internal Medicine, Mercer University ${ }^{2}, \mathrm{Macon}, \mathrm{OH}, \mathrm{USA}$
}

\begin{abstract}
Background/Aims: Inflammatory bowel disease (IBD) is a complex condition precipitated by genetic susceptibility and possibly a disturbed microbiome. The role of dairy foods in IBD is controversial. This study examined the association between lactose intolerance (LI) and IBD.

Methods: Data on hospital admissions of all IBD adult patients were extracted from the National Inpatient Sample database between 2004 and 2014. The comorbidities and outcomes of interest were defined by querying all the diagnostic and procedural fields for the corresponding International Classification of Diseases 9th version (ICD-9) codes. Patients with IBD were defined as the "study group," and the patients who did not have IBD were defined as the "control group". LI was identified in both groups using the ICD-9 codes. Multivariate logistic regression was performed to examine the association between IBD and LI.

Results: The total population was $71,342,237$ patients, of which $598,129(0.83 \%)$ had IBD. The IBD patients were younger (52 years vs. 57 years) and with fewer females (57.5\% vs. $60.1 \%)(p<0.001$ for all). After adjusting for the potential confounding factors, the IBD group had a significantly higher rate of $\mathrm{LI}$ (OR 2.71, 95\% $\mathrm{Cl} 2.55-2.88, \mathrm{p}<0.001)$ compared to the non-IBD group. The findings were similar on the further stratification of IBD into Crohn's disease compared to the control group (OR $2.70,95 \% \mathrm{Cl}$ 2.50-2.92, $\mathrm{p}<0.001$ ) and ulcerative colitis compared to the control group (OR 2.71, 95\% Cl 2.46-2.98, $\mathrm{p}<0.001$ ).

Conclusions: IBD patients have a 2.7 times higher risk of LI. Screening for LI in this population is warranted to avoid confusing or overlapping symptomatology. (Korean J Gastroenterol 2020;76:185-190)
\end{abstract}

Key Words: Lactose intolerance; Inflammatory bowel diseases; Colitis, ulcerative; Crohn disease; NIS

\section{INTRODUCTION}

Inflammatory bowel disease (IBD) is an umbrella term for two idiopathic chronic inflammatory diseases: ulcerative colitis (UC) and Crohn disease (CD). ${ }^{1}$ The incidence of IBD is increasing, particularly in Western countries. In North America, the incidence of UC was reported to be as high as 19.2 per 100,000 person-years, and as high as 20.2 per 100,000 person-years for $C D .^{2}$ The pathogenesis of IBD is unclear, but may be due to a dysregulation of the immune system in re- sponse to changes in the normal gut flora. ${ }^{3}$ Additionally, multiple factors have been attributed to IBD development as well such as genetics, infections, Western diet, and smoking. ${ }^{4}$

Lactose malabsorption is present in approximately $65-70 \%$ of the population, which causes symptoms that may overlap with the symptoms of IBD, including, but not limited to, diarrhea, abdominal pain, and bloating. ${ }^{5}$ Lactose intolerance (LI) is characterized by the inability to digest lactose disaccharides. ${ }^{6} \mathrm{LI}$ depends not only on the expression of lactase enzyme in the small intestines, but also on the dose of lactose, intestinal

Received April 1, 2020. Revised July 6, 2020. Accepted July 9, 2020.

(C) This is an open access article distributed under the terms of the Creative Commons Attribution Non-Commercial License (http://creativecommons.org/licenses/ by-nc/4.0) which permits unrestricted non-commercial use, distribution, and reproduction in any medium, provided the original work is properly cited. Copyright (c) 2020. Korean Society of Gastroenterology.

Correspondence to: Mohammad Maysara Asfari, Department of Gastroenterology, Medical College of Georgia/Augusta University, 1120 15th Street, Augusta, GA 30912, USA. Tel: +1-706-721-0207, Fax: +1-706-721-0331, E-mail: mma-86@hotmail.com, ORCID: https://orcid.org/0000-0003-0059-9538

Financial support: None. Conflict of interest: None. 
flora and bacterial overgrowth, gastrointestinal motility and sensitivity of the gastrointestinal tract to the generation of gas and other fermentation products of lactose malabsorption. ${ }^{7}$ Given the overlapping symptoms between IBD and LI, this study examined the association of $\mathrm{LI}$ with IBD using very large inpatient data.

\section{SUBJECTS AND METHODS}

\section{Patient population}

A cross-sectional study was conducted using the National Inpatient Sample (NIS) data from 2004 to 2014. The NIS is the largest all-payer inpatient database in the USA. The database contains a sample of more than eight million inpatient stays each year, representing approximately $20 \%$ sample of discharges from all community hospitals participating in the Healthcare Cost and Utilization Project. It does not include rehabilitation and long-term acute care hospitals. Each record of the NIS data includes primary and secondary diagnoses up to 25 and primary and secondary procedures up to 15. Furthermore, it also contains the patients' demographics, discharge status, length of stay, disease severity, and comorbidity measures.

2. Study population, inclusion, and exclusion criteria All adult patients ( $\geq 18$ years old) were included from the NIS data from 2004-2014. Using the International Classification of Diseases 9th version (ICD-9) code, all records with $\mathrm{LI}$ and IBD were identified using the following codes: 271.3 and 571.8 , respectively. The patient demographics and comorbidities were identified using the Clinical Classification Software codes provided by the Healthcare Cost and Utilization Project, Elixhauser comorbidities, and appropriate ICD-9 codes. Table 1 lists the
Elixhauser comorbidities, whereas Table 2 lists the ICD-9 codes used for LI, IBD, and other comorbidities of interest. Institutional Review Board approval was not required because NIS is a publicly available database.

The association between IBD and LI was assessed by dividing the patients into two groups: patients with IBD (study group) and patients without IBD (control group). Multivariable logistic regression analysis was performed to compare the two groups after adjusting for any potential confounding factors. Similarly, subgroup analysis was performed to assess the association between both $\mathrm{CD}$ and UC with $\mathrm{LI}$.

\section{Statistical Analysis}

The data are expressed as mean values $\pm \mathrm{SD}$, and the frequencies were reported accordingly in percentages. Independent $t$-tests were used to compare the continuous variables, and a chi-square test was used for categorical variables. Multiple logistic regression was used to assess the association between $\mathrm{LI}$ and IBD. The regression model was adjusted for the patients' demographics, other relevant comorbidities (obesity, smoking, and alcohol abuse), hospital characteristics, patient insurance, and socioeconomic status. p-values less than 0.05 were considered significant. SPSS version 25 software (IBM Corp, Armonk, NY, USA) was used for all statistical analyses. The data in this study were obtained from pre-existing national data and did not require approval from an Institutional Review Board.

\section{RESULTS}

The nationwide cohort evaluated a total of 71,342,237 patients, of which 598,129 (0.83\%) had IBD and 57,909 $(0.08 \%)$ had LI. The patients with IBD were younger (52 years vs. 57 years), with comparatively fewer females (57.5\% vs.

Table 1. List of Elixhauser Comorbidities Included in the Analysis

\begin{tabular}{lcc}
\hline Obesity & ICD-9-CM \\
\hline ICD-9-CM, International Classification of Diseases, ninth edition, clinical modification. & \\
& & \\
Table 2. ICD-9-CM, Clinical Modification, and CCS Codes used to Identify Comorbidities, Procedures, and Outcomes & Source \\
\hline Variables & CCS & Code(s) \\
\hline Inflammatory bowel disease & ICD-9-CM \\
Lactose intolerance & ICD-9-CM & 271.3 \\
Smoking & 305.1
\end{tabular}

ICD-9-CM, International Classification of Diseases, ninth edition, clinical modification; CCS, Clinical Classification Software. 
$60.1 \%)$ and fewer African Americans (10\% vs. $14.3 \%)$ compared to the patients without IBD ( $p<0.001$ for all). In addition, the patients in the IBD group had a higher prevalence of smoking $(14 \%$ vs. $12 \%)(p<0.001)$ compared to the patients without IBD. On the other hand, patients in the study group were less obese $(6.7 \%$ vs. $8.9 \%)(p<0.001)$ compared to the control group (details are shown in Table 3).

Multivariable logistic regression was performed after adjusting for the possible confounding factors, including age, gender, race, patient demographics, hospital characteristics,

Table 3. Comparison of Inflammatory Bowel Disease and Non-inflammatory Bowel Disease Patients

\begin{tabular}{|c|c|c|c|}
\hline Variables & IBD $(n=598,129)$ & Non-IBD $(n=70,744,108)$ & p-value \\
\hline Age (years) & $51.97 \pm 19.12$ & $57.09 \pm 20.86$ & $<0.001$ \\
\hline Sex & & & $<0.001$ \\
\hline Female & 57.5 & 59.6 & \\
\hline Race & & & $<0.001$ \\
\hline White & 81.1 & 68.8 & \\
\hline Black & 10.0 & 14.3 & \\
\hline Hispanic & 5.3 & 10.8 & \\
\hline Asian or Pacific Islander & 1.0 & 2.4 & \\
\hline Native American & 0.4 & 0.6 & \\
\hline Other & 2.3 & 3.0 & \\
\hline Primary expected payer & & & $<0.001$ \\
\hline Medicare & 37.6 & 45.3 & \\
\hline Medicaid & 11.1 & 15.2 & \\
\hline Private insurance & 42.6 & 30.5 & \\
\hline Self-pay & 4.9 & 5.3 & \\
\hline No charge & 0.6 & 0.5 & \\
\hline Other & 3.2 & 3.3 & \\
\hline Median household income & & & $<0.001$ \\
\hline 0 to 25 & 23.0 & 29.3 & \\
\hline 26 to 50 & 25.2 & 26.1 & \\
\hline 51 to 75 & 23.3 & 23.6 & \\
\hline 76 to 100 & 21.0 & 21.0 & \\
\hline Bed size & & & $<0.001$ \\
\hline Small & 12.9 & 13.7 & \\
\hline Medium & 24.5 & 25.1 & \\
\hline Large & 62.6 & 61.2 & \\
\hline Location/teaching status & & & $<0.001$ \\
\hline Rural & 10.4 & 12.4 & \\
\hline Urban nonteaching & 38.7 & 41.1 & \\
\hline Urban teaching & 50.9 & 46.5 & \\
\hline Hospital region & & & $<0.001$ \\
\hline Northeast & 22.3 & 19.4 & \\
\hline Midwest & 25.2 & 22.7 & \\
\hline South & 35.8 & 39.0 & \\
\hline West & 16.7 & 18.9 & \\
\hline Obesity & 6.7 & 8.9 & $<0.001$ \\
\hline Smoking & 14.0 & 12.0 & $<0.001$ \\
\hline
\end{tabular}

Values are presented as mean \pm standard deviation or \%.

$\mathrm{IBD}$, inflammatory bowel disease; SD, standard deviation. 


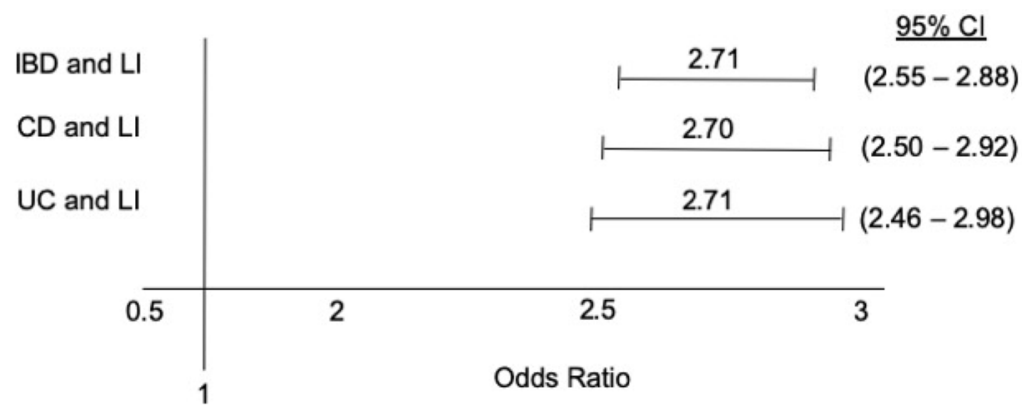

Fig. 1. Association of IBD with LI. IBD, inflammatory bowel disease; CD, Crohn's disease; UC, ulcerative colitis; LI, lactose intolerance; $\mathrm{Cl}$, confidence interval.

patient insurance and socioeconomic status, and other relevant comorbidities (obesity and smoking). The results showed that patients with IBD had higher odds of having LI (OR 2.71, $95 \% \mathrm{Cl} 2.55-2.88, \mathrm{p}<0.001)$. Similarly, patients with CD and UC had a higher odds of LI: (OR 2.70, 95\% Cl 2.50-2.92) and (OR 2.71, 95\% Cl 2.46-2.98), respectively ( $p<0.001$ for all) (forest plot is shown in Fig. 1).

\section{DISCUSSION}

Functional Gl symptoms, such as abdominal pain, distention, gas, bloating, and alterations of the bowel habits, are commonly observed among patients with IBD, irritable bowel syndrome, dietary intolerance, small intestinal bacterial overgrowth, bile salt diarrhea, and other functional GI disorders. ${ }^{8}$ These functional Gl symptoms may overlap with the symptoms associated with IBD-related inflammation despite achieving endoscopic and histologic mucosal healing. ${ }^{9}$ When objective evidence of active inflammation does not correlate with the persistent symptoms in IBD patients, clinicians should consider other diseases with alternate pathophysiological mechanisms, presenting with functional Gl symptoms. Differentiating the symptoms of IBD-related inflammatory changes from symptoms of non-IBD diseases is important to avoid the overtreatment of IBD-related intestinal inflammation, given its associated adverse effects. ${ }^{9}$

$\mathrm{LI}$ is a condition with an inability to digest or absorb lactose disaccharide and may occur in IBD patients because of the resulting changes in the structure and function of the Gl tract related to the chronic inflammatory state of IBD. ${ }^{8}$ It is largely due to loss of the enzyme lactase phlorizin hydrolase (LPH) in the gut. The incidence of $\mathrm{LI}$ increases with the age with onset from 2-12 years. ${ }^{10}$ The mechanism causing the loss of lactase enzyme is unknown, even though recent studies have shown that random mutations upstream of the LCT gene could be a contributing factor. ${ }^{11}$ Owing to the inability to digest lactose, it accumulates in the gut, which is then digested by the colonic bacteria, yielding methane and other metabolites, causing symptoms related to luminal distention and stimulation of mechanoreceptors. ${ }^{12}$ Patients with IBD with concomitant LI may present with persistent Gl symptoms, such as abdominal pain, bloating, fullness, and diarrhea, giving a false impression of active IBD despite the quiescent disease. ${ }^{13}$

Based on this analysis, both UC and CD patients had a significantly higher risk of having $\mathrm{LI}$ after adjusting for the possible confounding factors, including age, race, gender, obesity, and tobacco use. This study confirmed the findings of the largest meta-analysis by Szilagyi et al. ${ }^{14}$, which included 17 studies and 1,935 IBD patients. The risk of lactose maldigestion was reported to be higher among IBD patients than the controls. In subgroup analysis of this meta-analysis, the risk of lactose maldigestion was only significant for CD but not UC patients compared to another study, wherein both CD and UC patients were at a significantly higher risk of LI. In the 1990s, Mishkin reported that LI prevalence in UC patients was more likely to follow the normal population, whereas $C D$ patients had prevalence beyond the ethnic risk. ${ }^{15}$ The additional risk in $\mathrm{CD}$ and $\mathrm{LI}$ correlated with the ethnic risk, location of the disease (with the small bowel having the highest risk), and surgery. ${ }^{16}$ Mishkin et al. reported that patients with $C D$ and lactose malabsorption, who were identified using breath-hydrogen tests, have a shorter transit time of the small bowel. ${ }^{15,17}$ In the active phases of the disease, both $U C$ and CD patients showed improved symptoms after being given lactose-free diets. ${ }^{18}$ In UC, alterations of the small bowel mucosa may cause decreases in the disaccharidase content in the epi- 
thelium, and thus a decreased lactase enzyme level. ${ }^{5}$ Eadala et al. $^{13}$, in their examination of 165 IBD patients with CC13910 genotype, reported that sensitivity to lactose occurs in a high percentage of patients in remission. They highlighted the importance of a thorough consideration of lactose malabsorption in IBD patients because of their similar symptomatology.

The hydrogen breath test remains the most common method of diagnosing lactose intolerance worldwide. ${ }^{19}$ Although a jejunal biopsy is the most accurate test for LI, its use is limited by its invasiveness. ${ }^{20}$ Genetic testing is a promising test, but its use is not sensitive in non-Caucasians and may not be suitable for assessing the secondary causes of LI. ${ }^{11,20}$ Owing to the limitations of $\mathrm{LI}$ diagnostic testing, a detailed history is the key to diagnosing $\mathrm{LI}$, particularly in patients with IBD. Developing more reliable testing for $\mathrm{LI}$ is required because a $\mathrm{LI}$ diagnosis can be challenging in real practice.

Patients with CD may be inadvertently advised to follow dietary restrictions, such as lactose-reduced, fructose or fructan-reduced, fermentable oligosaccharides, disaccharides, monosaccharides, and polyols (FODMAP)-reduced, and gluten-free diets to help improve the functional symptoms. ${ }^{12}$ Many IBD patients are already undernourished, especially those with uncontrolled disease or in acute flares; restrictive diets can further worsen their malnutrition. Avoiding dairy products and its associated long-term effects on bone density contributes to the increasing rates of osteoporosis among IBD patients, who are already at an increased risk, particularly those on chronic steroid or immunosuppressants. ${ }^{14}$ Therefore, it is important to evaluate IBD patients for LI before subjecting them to a lactose-restricted diet, given its potential adverse effects.

To the best of the authors' knowledge, this the largest cross-sectional study that evaluated the association between $\mathrm{LI}$ and IBD in a large segment of the United States population. This study had some limitations. Owing to ICD 9 limitations, NIS cannot specify if the diagnosis of LI was proven by testing. In addition, NIS relies on the accuracy of clinical data and the validity of medical diagnoses, which might differ among individuals and facilities. Furthermore, NIS cannot specify the severity of the IBD disease. Because NIS is based on inpatient data, this inclusion could lead to a larger number of sick individuals in the data, which might have affected the generalizability of the results. The exclusion of academic hospitals by the database could potentially exclude patients with more complex diseases. Regardless of these limitations, this study revealed a strong association between IBD and $\mathrm{LI}$ and highlighted the importance of $\mathrm{LI}$ screening in IBD patients. $\mathrm{LI}$ is a commonly observed finding among adults and is more common in those who suffer from both UC and CD. Screening for LI should be considered in all IBD patients to help identify the etiology of their Gl symptoms and potentially avoid unnecessary tests or changes in their maintaining regimens.

\section{REFERENCES}

1. Kappelman MD, Moore KR, Allen JK, Cook SF. Recent trends in the prevalence of Crohn's disease and ulcerative colitis in a commercially insured us population. Dig Dis Sci 2013;58:519-525.

2. M'Koma AE. Inflammatory bowel disease : an expanding global health problem. Clin Med Insights Gastroenterol 2013;6:33-47.

3. Sairenji T, Collins KL, Evans DV. An update on inflammatory bowel disease. Prim Care 2017;44:673-692.

4. Ananthakrishnan A, Bernstein C, lliopoulos D, et al. Environmental triggers in IBD: a review of progress and evidence. Nat Rev Gastroenterol Hepatol 2018;15:39-49.

5. Gudmand-Hoyer E, Jarnum S. Incidence and clinical significance of lactose malabsorption in ulcerative colitis and Crohn's disease. Gut 1970;11:338-343.

6. Kirschner BS, DeFavaro MV, Jensen W. Lactose malabsorption in children and adolescents with inflammatory bowel disease. Gastroenterology 1981;81:829-832.

7. Deng Y, Misselwitz B, Dai N, Fox M. Lactose intolerance in adults: biological mechanism and dietary management. Nutrients 2015;7:8020-8035.

8. Barrett JS, Irving PM, Shepherd SJ, Muir JG, Gibson PR. Comparison of the prevalence of fructose and lactose malabsorption across chronic intestinal disorders. Aliment Pharmacol Ther 2009;30:165-174.

9. Peyrin-Biroulet L, Reinisch W, Colombel JF, et al. Clinical disease activity, C-reactive protein normalisation and mucosal healing in Crohn's disease in the SONIC trial. Gut 2014;63:88-95.

10. Mishkin, S. Dairy sensitivity, lactose malabsorption, and elimination diets in inflammatory bowel disease. Am J Clin Nutr 1997;65:564-567.

11. Mattar R, de Campos Mazo DF, Carrilho FJ. Lactose intolerance: diagnosis, genetic, and clinical factors. Clin Exp Gastroenterol 2012;5:113-121.

12. Halmos EP, Christophersen CT, Bird AR, Shepherd SJ, Muir JG, Gibson PR. Consistent prebiotic effect on gut microbiota with altered FODMAP intake in patients with Crohn's disease: a randomised, controlled cross-over trial of well-defined diets. Clin Transl Gastroenterol 2016;7:e164.

13. Eadala P, Matthews SB, Waud JP, Green JT, Campbell AK. Association of lactose sensitivity with inflammatory bowel disease--demonstrated by analysis of genetic polymorphism, breath gases and symptoms. Aliment Pharmacol Ther 2011;34: 735-746. 
14. Szilagyi A, Galiatsatos P, Xue X. Systematic review and meta-analysis of lactose digestion, its impact on intolerance and nutritional effects of dairy food restriction in inflammatory bowel diseases. Nutr J 2016;15:67.

15. Mishkin S. Dairy sensitivity, lactose malabsorption, and elimination diets in inflammatory bowel disease. Am J Clin Nutr 1997;65:564-567.

16. Mishkin B, Yalovsky M, Mishkin S. Increased prevalence of lactose malabsorption in Crohn's disease patients at low risk for lactose malabsorption based on ethnic origin. Am J Gastroenterol 1997;92:1148-1153.

17. Hüppe D, Tromm A, Langhorst H, May B. Lactose intolerance in chronic inflammatory bowel diseases. Dtsch Med Wochenschr 1992;117:1550-1555.

18. Wright R, Truelove SC. A controlled therapeutic trial of various diets in ulcerative colitis. Br Med J 1965;2:138-141.

19. Furnari M, Bonfanti D, Parodi A, et al. A comparison between lactose breath test and quick test on duodenal biopsies for diagnosing lactase deficiency in patients with self-reported lactose intolerance. J Clin Gastroenterol 2013;47:148-152.

20. Misselwitz B, Pohl D, Frühauf H, Fried M, Vavricka SR, Fox M. Lactose malabsorption and intolerance: pathogenesis, diagnosis and treatment. United European Gastroenterol J 2013;1: 151-159. 